\title{
Targeting of NHERF1 through RNA interference inhibits the proliferation and migration of metastatic prostate cancer cells
}

\author{
QIANG MA $^{1}$, YANNA JIAO ${ }^{2}$, YUMEI HAO ${ }^{1}$, SHANSHAN YAN ${ }^{3}$, NENAN LYU $^{4}$, \\ HONGLIN GAO ${ }^{1}$, DEGUAN LI ${ }^{1}$, QIANG LIU ${ }^{1}$, JUNFANG ZHENG ${ }^{5}$ and NALING SONG ${ }^{1}$
}

\begin{abstract}
${ }^{1}$ Tianjin Key Laboratory of Radiation Medicine and Molecular Nuclear Medicine, Institute of Radiation Medicine, Peking Union Medical College and Chinese Academy of Medical Science, Tianjin 300192; ${ }^{2}$ Department of Genetics, National Research Institute for Family Planning, Beijing 100081; ${ }^{3}$ Department of Molecular Biology, Institute of Medical Biology, Peking Union Medical College and Chinese Academy of Medical Science, Kunming, Yunnan 650000; ${ }^{4}$ Department of Gynecologic Oncology, Beijing Obstetrics and Gynecology Hospital, Capital Medical University, Beijing 100026; ${ }^{5}$ Department of Biochemistry and Molecular Biology, Capital Medical University, Beijing 100069, P.R. China
\end{abstract}

Received August 22, 2014; Accepted May 8, 2015

DOI: $10.3892 / \mathrm{ol} .2015 .4007$

\begin{abstract}
The present study aimed to investigate the effects of $\mathrm{Na}^{+} / \mathrm{H}^{+}$exchanger regulatory factor 1 (NHERF1) gene knockdown, using short-hairpin RNA (shRNA), on the malignant behaviors of prostate cancer cells. A pSuper.puro NHERF1 shRNA vector was transfected into PC-3M prostate cancer cells using Lipofectamine 2000. Stable cell lines were obtained and NHERF1 knockdown was verified through western blot analysis. MTT assays were then used to measure PC-3M cell proliferation; in addition, cell migration was assessed using a wound healing assay. Flow cytometry was employed in order to determine the effects of NHERF1 knockdown on apoptosis. Expression levels of apoptotic pathway proteins B cell lymphoma-2 (Bcl-2) and Bcl-2-associated X protein were then determined by western blot analysis. The results demonstrated that shRNA knockdown of NHERF1 significantly suppressed the proliferation of $\mathrm{PC}-3 \mathrm{M}$ cells by $>50 \%$. In addition, knockdown of NHERF1 significantly inhibited the migration of PC-3M cells. PC-3M cells harboring NHERF1 shRNA exhibited significantly increased apoptosis, with an $\sim$-fold increase compared with
\end{abstract}

Correspondence to: Professor Naling Song, Tianjin Key Laboratory of Radiation Medicine and Molecular Nuclear Medicine, Institute of Radiation Medicine, Peking Union Medical College and Chinese Academy of Medical Science, Room 319, Administrative Building, 238 Baidi Road, Nankai, Tianjin 300192, P.R. China E-mail: nalingsong@sina.com

Dr Junfang Zheng, Department of Biochemistry and Molecular Biology, Capital Medical University, Room 1019, Basic Research Building, 10 Xitoutiao, You An Men, Fengtai, Beijing 100069, P.R. China

E-mail: zhengjf@ccmu.edu.cn

Key words: prostate cancer, $\mathrm{Na}^{+} / \mathrm{H}^{+}$exchanger regulatory factor 1 , apoptosis that of the parental PC-3M cells and cells transfected with an empty vector. Furthermore, the results revealed that knockdown of NHERF1 reduced the protein expression of $\mathrm{Bcl}-2$, although the expression of Bax was unaltered. In conclusion, NHERF1 knockdown using shRNA inhibited the proliferation and migration of PC-3M cells and promoted apoptosis, highlighting the role of NHERF1 in prostate cancer progression.

\section{Introduction}

Prostate cancer is the most prevalent type of tumor of the male urinary system. In the United States, prostate cancer is the second leading cause of cancer in men, the incidence of which is rising in China as well as in Western countries (1). In general, prostate cancer is a slow-growing disease, with the onset of symptoms occurring in advanced-stage disease, following tumor discovery (2). The initial treatment for prostate cancer is usually hormone therapy; however, when prostate tumors progress into an androgen-independent state, treatment becomes difficult (1).

$\mathrm{Na}^{+} / \mathrm{H}^{+}$exchanger regulatory factor 1 (NHERF1) was identified to be a multifunctional scaffolding protein that has various functions in numerous types of cancer through its interactions with oncogenic or tumor-suppressor proteins (3). In breast cancer, NHERF1 acts as a tumor suppressor protein, which is involved in influencing signal transduction and modulating the expression of phosphatase and tensin homolog (PTEN) to regulate the malignant phenotype (4). However, NHERF1 has been shown to have oncogenic functions in glioma and other types of cancer (5). While the specific role of NHERF1 in prostate cancer remains to be elucidated, studies have shown that the NHERF1 protein was overexpressed in tumor specimens in situ compared with normal prostate tissues $(6,7)$. Therefore, further studies are required in order to fully elucidate the role of NHERF1 in prostate cancer.

The present study aimed to determine the effects of NHREF1 knockdown using short-hairpin RNA (shRNA) in 
PC-3M cells, a prostate cancer cell line exhibiting abnormally high expression of NHERF1.

\section{Materials and methods}

Bacterial strains, cell lines and plasmids. The bacterial strain Escherichia coli DH5 $\alpha$ was purchased from Beijing CoWin Biotech Co., Ltd. (Beijing, China). The pSuper.puro NHERF1 shRNA plasmid and negative control plasmid (pSuper. puro luciferase shRNA) were a gift from Professor Junfang Zheng (Capital Medical University, Beijing, China), originally constructed by Dr Margaret J. Wheelock (University of Nebraska Medical Center, Omaha, NE, USA). PC-3M prostate cancer cells were purchased from the Cell Resource Center of Beijing Xiehe (Beijing, China).

Reagents. RPMI 1640 medium, fetal calf serum and Lipofectamine 2000 were purchased from Invitrogen Life Technologies (Carlsbad, CA, USA). Rabbit anti-human NHERF1 (cat no. ab133599) and Bcl-2-associated X protein (Bax; cat no. ab32503) monoclonal antibodies, and rabbit anti-human GADPH (cat no. ab181602) polyclonal antibody were purchased from Abcam (Cambridge, UK). Rabbit anti-human B cell lymphoma-2 (Bcl-2; cat no. ZA-0536) monoclonal antibody was purchased from Beijing Zhongshan GoldenBridge Biotechnology Co., Ltd. (Beijing, China), and goat anti-rabbit horseradish peroxidase (HRP)-conjugated secondary antibody (cat no. CW0103) was purchased from Beijing CoWin Biotech Co., Ltd. MTT was purchased from Sigma-Aldrich (St. Louis, MO, USA). The Annexin V-FITC Apoptosis Detection kit and Propidium Staining Cycle Detection kit were obtained from BestBio Biotechnology Co., Ltd. (Shanghai, China). All other reagents were of analytic grade.

Conversion and amplification of plasmid. The interference plasmid pSuper.puro harboring NHERF1 shRNA and the negative control plasmid pSuper.puro harboring luciferase shRNA were transformed into competent $E$. coli $\mathrm{DH} 5 \alpha$ cells. Briefly, competent E. coli DH5 $\alpha(100 \mu \mathrm{l})$ and plasmid $(2 \mu \mathrm{l})$ were added to Eppendorf tubes, and placed in ice for $30 \mathrm{~min}$. Next, the Eppendorf tubes were incubated at $42^{\circ} \mathrm{C}$ for $90 \mathrm{sec}$ and then placed back in ice for $2 \mathrm{~min}$. Lysogeny broth (LB) media without antibiotic (800 $\mu \mathrm{l}$; Thermo Fisher Scientific, Waltham, MA, USA) was added and the transformed DH5 $\alpha$ cells were incubated at $37^{\circ} \mathrm{C}$ for 30 min with agitation. Transformed DH5 $\alpha(50 \mu \mathrm{l})$ were spread on LB culture plates (Corning Incorporated, Corning, NY, USA) containing ampicillin and cultured at $37^{\circ} \mathrm{C}$ for $12-16 \mathrm{~h}$. Positive colonies were selected following cultivation of the bacteria on LB plates (Beijing CoWin Biotech Co., Ltd.) containing $100 \mu \mathrm{g} / \mathrm{ml}$ ampicillin (Sigma-Aldrich); the plasmid was then extracted and purified following overnight cultivation of the bacteria.

Cell culture and the establishment of a stable expression system. PC-3M cells were cultured in RPMI 1640 medium containing $10 \%$ fetal calf serum and $1 \%$ penicillin-streptomycin (Sigma-Aldrich) at $37^{\circ} \mathrm{C}$ in an incubator with $5 \%$ $\mathrm{CO}_{2}$. Cells were transfected with $2.5 \mu \mathrm{g}$ plasmid using Lipofectamine 2000, according to the manufacturer's instructions. Stably transfected cells were selected using $2 \mu \mathrm{g} / \mathrm{ml}$ puromycin
(Sigma-Aldrich) for 3-4 weeks at $37^{\circ} \mathrm{C}$ in an incubator with 5\% $\mathrm{CO}_{2}$. Clones were maintained in culture medium containing $1 \mu \mathrm{g} / \mathrm{ml}$ puromycin. Media were replaced every 3 days.

Detection of cell growth. Cells stably expressing NHERF1 shRNA or negative control shRNA were plated at a density of $5 \times 10^{3}$ cells/well onto 96 -well plates at $37^{\circ} \mathrm{C}$ in an incubator with $5 \% \mathrm{CO}_{2}$. Cell proliferation was then assessed every $24 \mathrm{~h}$ for $96 \mathrm{~h}$ using MTT assays according to standard protocols (8). For each sample at each time-point, six wells were analyzed. The experiment was repeated three times.

Western blotting. Cells were collected and lysed in radioimmunoprecipitation buffer (Beijing CoWin Biotech Co., Ltd., Beijing, China) in the presence of protease inhibitors for $20 \mathrm{~min}$ to extract total protein from cells stably expressing NHERFI shRNA or control cells, and protein levels were quantified using bicinchoninic acid assays (Beijing CoWin Biotech Co., Ltd.). Subsequently, $50 \mu \mathrm{g}$ protein from each sample was loaded onto 8\% sodium dodecyl sulfate (SDS) polyacrylamide gels and subjected to SDS-polyacrylamide gel electrophoresis (PAGE; Beijing CoWin Biotech Co., Ltd.). Protein was then transferred to nitrocellulose membranes (Sigma-Aldrich), which were then blocked with bovine serum albumin blocking buffer (Invitrogen Life Technologies) for $1 \mathrm{~h}$. Membranes were then incubated with primary antibodies targeting NHERF1 (1:1,000 dilution), Bcl-2 (1:1,000 dilution), Bax (1:200 dilution) or GAPDH (1:1,000 dilution) overnight at $4^{\circ} \mathrm{C}$, followed by incubation with secondary antibodies conjugated with HRP (1:3,000 dilution) for $1 \mathrm{~h}$ at room temperature. Detection was facilitated using an enhanced chemiluminescence kit and images were analyzed using ImageJ software (version 1.62; National Institute of Health, Bethesda, MD, USA).

Wound healing assay. Cells were plated at a density of $2 \times 10^{5}$ cells/well onto six-well plates and grown to $80 \%$ confluence. The monolayer of cells was scratched with a 200- $\mu 1$ pipette tip to create a wound and an image was captured immediately $(0 \mathrm{~h})$. Migration was then observed every $12 \mathrm{~h}$ and images of the closing wound were captured at each time-point with a digital camera connected to a phase-contrast microscope (CKX41; 10x objective lens; Olympus, Tokyo, Japan). The same visual field was used throughout the experiment. Image-Pro Plus software (version 6.0; Media Cybernetics, Rockville, MD, USA) was used to facilitate the calculation of the migration rate as follows: Migration rate $=[$ (relative distance recorded at $0 \mathrm{~h}$ - relative distance recorded at $48 \mathrm{~h}$ ) / relative distance recorded at $0 \mathrm{~h}] \times 100 \%$. The experiment was repeated three times.

Analysis of cell apoptosis. Annexin V-fluorescein isothiocyanate (FITC)/propidium iodide (PI) flow cytometry was used to detect the rate of apoptosis. Cells were collected, washed with phosphate-buffer saline (PBS) and resuspended in $400 \mu 1$ buffer (BestBio Biotechnology Co., Ltd.). Subsequently, $5 \mu 1$ Annexin V-FITC (BestBio Biotechnology Co., Ltd.) was added, cells were mixed and the solution was incubated for $15 \mathrm{~min}$ in the dark at room temperature. PI (10 $\mu \mathrm{l}$; BestBio Biotechnology Co., Ltd.) was then added and the samples 


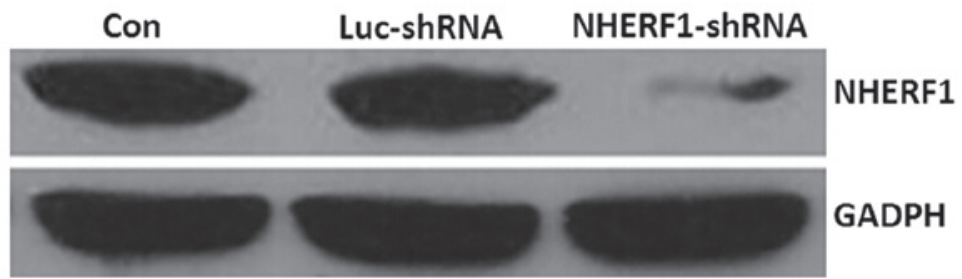

Figure 1. Expression of NHERF1 in PC-3M cells transfected with NHERF1-targeting shRNA. Western blot analysis was used to detect NHERF1 expression in Con, Luc-shRNA and NHERF1-shRNA cells. GAPDH was used as a loading control. NHERF1, $\mathrm{Na}^{+} / \mathrm{H}^{+}$exchanger regulatory factor 1; shRNA, short-hairpin RNA; Con, untransfected cells; Luc-shRNA, cells stably transfected with luciferase shRNA; NHERF1-shRNA, cells stably transfected with NHERF1 shRNA.

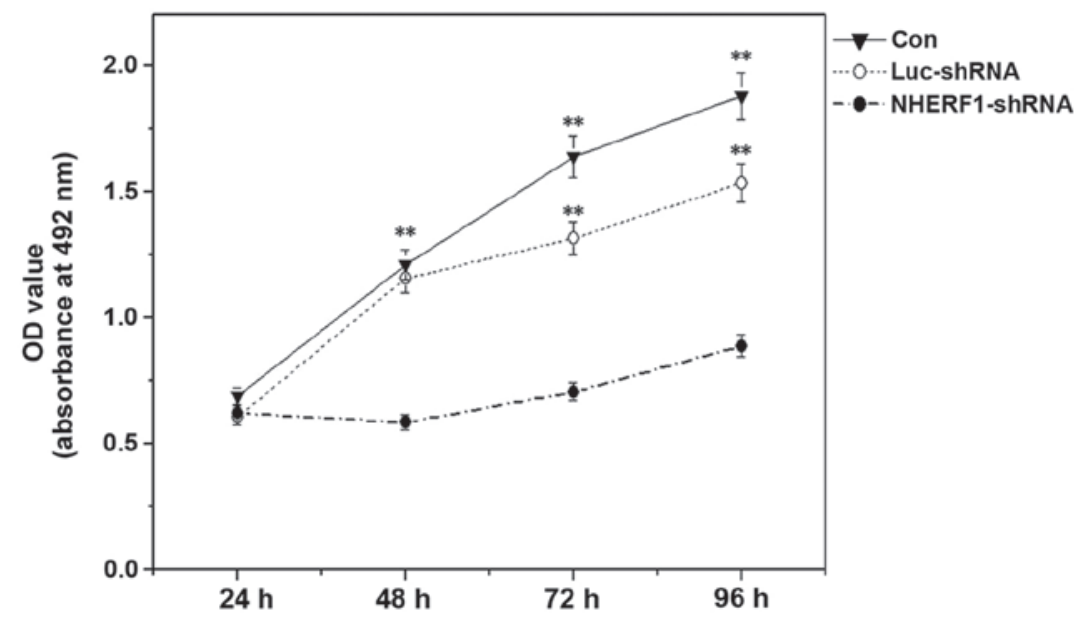

Figure 2. Growth curves for PC-3M cells stably expressing NHERF1 shRNA. Cell proliferation was measured every $24 \mathrm{~h}$ up to $96 \mathrm{~h}$ in Con, Luc-shRNA and NHERF1-shRNA cells using an MTT assay. ${ }^{* *} \mathrm{P}<0.01$ vs. Con and Luc-shRNA. NHERF1; $\mathrm{Na}^{+} / \mathrm{H}^{+}$exchanger regulatory factor 1 ; shRNA, short-hairpin RNA; OD, optical density; Con, untransfected cells; Luc-shRNA, cells stably transfected with luciferase shRNA; NHERF1-shRNA, cells stably transfected with NHERF1 shRNA.
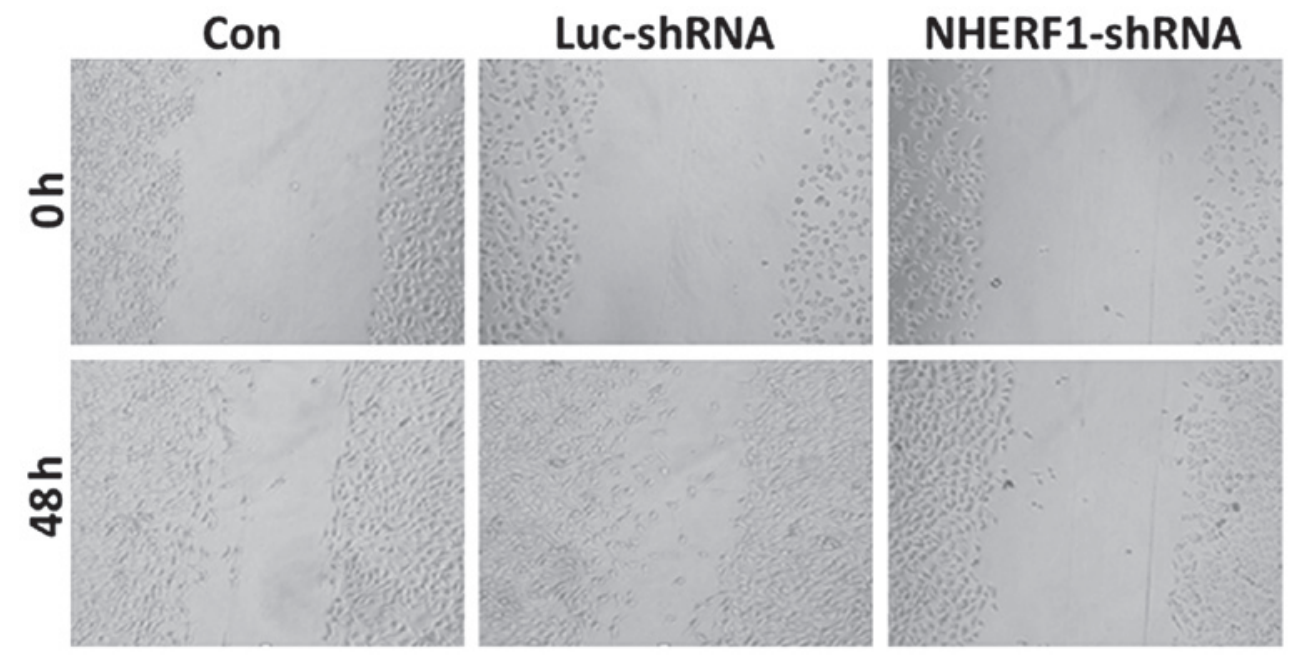

Figure 3. Migration of PC-3M cells transfected with NHERF1 shRNA. Wound healing assays were performed using Con,Luc-shRNA and NHERF1-shRNA cells in order to determine the rate of migration following $48 \mathrm{~h}$. NHERF1; $\mathrm{Na}^{+} / \mathrm{H}^{+}$exchanger regulatory factor 1; shRNA, short-hairpin RNA; Con, untransfected cells; Luc-shRNA, cells stably transfected with luciferase shRNA; NHERF1-shRNA, cells stably transfected with NHERF1 shRNA.

were incubated in the dark at room temperature for $5 \mathrm{~min}$. Cells were then analyzed using flow cytometry within $1 \mathrm{~h}$ (Accuri C6; BD Biosciences, Franklin Lakes, NJ, USA).

Analysis of cell cycle. Cultured cells were removed with trypsin and fixed with $70 \%$ ethanol at $4^{\circ} \mathrm{C}$ overnight. Subsequently, they were stained with PI ( $20 \mu \mathrm{g} / \mathrm{ml}$ PI, $200 \mu \mathrm{g} / \mathrm{ml}$ DNase-free RNase A and $0.1 \%$ Triton X-100, prepared freshly in PBS). The cellular DNA content was analyzed using a FACSCalibur machine (Becton Dickinson, San Jose, CA, USA). Data were analyzed using CellQuest software (version 3.0; Becton Dickinson.) 

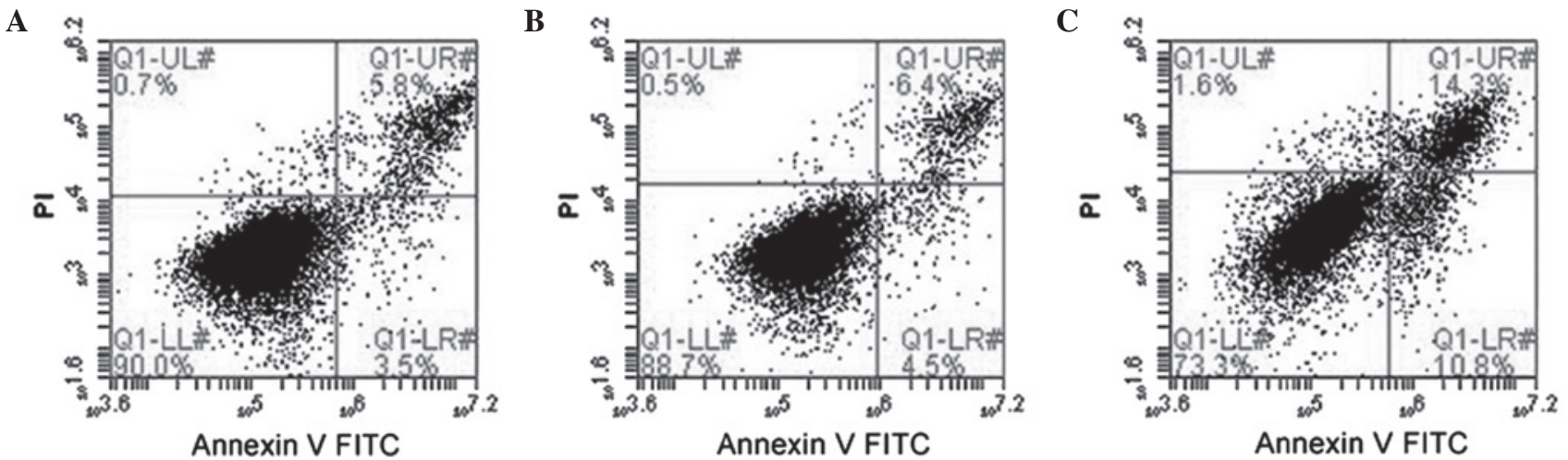

Figure 4. Flow cytometry analysis of apoptosis in PC-3M cells. Double staining with Annexin V-FITC and PI was used to assess apoptosis in (A) untransfected cells, (B) cells transfected with luciferase shRNA and (C) cells transfected with NHERF1 shRNA. FITC, fluorescein isothiocyanate; PI, propidium iodide; NHERF1; $\mathrm{Na}^{+} / \mathrm{H}^{+}$exchanger regulatory factor 1 ; shRNA, short-hairpin RNA.

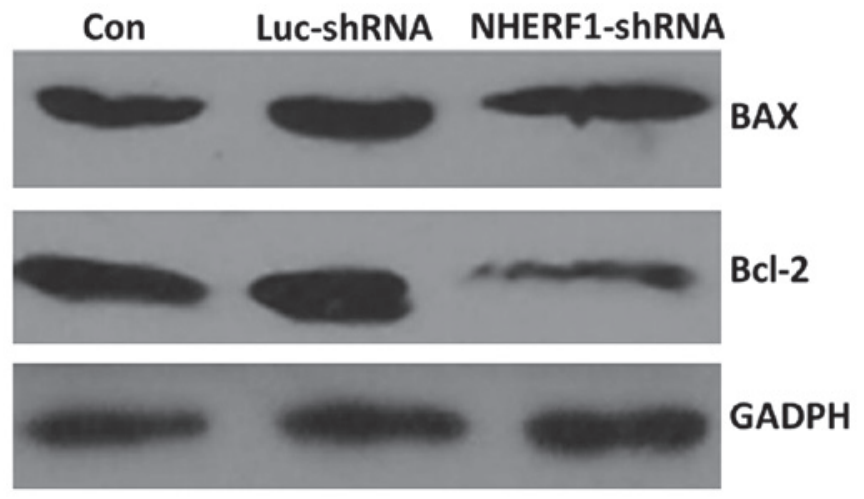

Figure 5.Expression of Bcl-2 and Bax in PC-3M cells transfected with NHERF1 shRNA. Western blot analysis was performed to determine Bcl-2 and Bax protein expression in Con, Luc-shRNA and NHERF1-shRNA cells. Data are representative of three identical experiments. Bcl-2, B cell lymphoma 2; Bax, Bcl-2-associated X protein; $\mathrm{NHERF} 1 ; \mathrm{Na}^{+} / \mathrm{H}^{+}$exchanger regulatory factor 1 ; shRNA, short-hairpin RNA; Con, untransfected cells; Luc-shRNA, cells stably transfected with luciferase shRNA; NHERF1-shRNA, cells stably transfected with NHERF1 shRNA.

Statistical analysis. All experiments were repeated at least three times. SPSS 11.5 software (SPSS, Inc., Chicago, IL, USA) was used to analyze the results. Growth curves were analyzed using a repeated-measures analysis of variance with Fisher's least significant difference post-hoc tests and apoptosis was analyzed using independent sample t-tests. $\mathrm{P}<0.05$ was considered to indicate a statistically significant difference between values.

\section{Results}

Analysis of NHERF1 knockdown in stably transfected $P C-3 M$ cells. As shown in Fig. 1, NHERF1 expression was successfully knocked down in NHERF1 shRNA-transfected cells compared with untransfected cells and cells transfected with the negative control plasmid. These cells were used in subsequent experiments as NHERF1 knockdown cells.

Influence of NHERF1 knockdown on the proliferation capacity of PC-3M cells. The ability of NHERF1 to modulate the proliferation of prostate cancer cells was analyzed using an MTT assay. The results indicated that NHERF1 knockdown significantly inhibited the proliferation capacity of PC-3M cells by $>50 \%$ at all time-points following $24 \mathrm{~h}$ of incubation, as compared with control cells $(\mathrm{P}<0.01)$ (Fig. 2). No significant differences were observed between untransfected cells and luciferase shRNA-transfected cells.

Influence of NHERF1 knockdown on the migration capacity of $P C$-3M cells. At $48 \mathrm{~h}$ following wounding, there were no significant differences in wound healing rates between untransfected cells and cells transfected with luciferase shRNA (Fig. 3). However, cells transfected with NHERF1 shRNA exhibited a significant reduction in migration capacity $(\mathrm{P}<0.05)$, with the wound size measuring $94-98 \%$ of the wound size at $0 \mathrm{~h}$ (Fig. 3). Thus, these data demonstrated that knockdown of NHERF1 significantly inhibited the migration capacity of PC-3M prostate cancer cells.

Influence of NHERF1 knockdown on apoptosis in PC-3M cells. Representative results of flow cytometric analysis are shown in Fig. 4. In general, the rates of cell death were low $(<5 \%)$. However, cells stably expressing NHREF1 shRNA exhibited a significant increase in apoptosis by $\sim 4$-fold compared with cells transfected with luciferase shRNA (11.98 \pm 3.28 vs. $2.55 \pm 0.92 \%$, respectively; $\mathrm{P}<0.001$ ); untransfected cells exhibited $3.5 \pm 0.96 \%$ apoptosis compared with the luciferase shRNA-transfected cells $(\mathrm{P}>0.05)$. These data indicated that reduced NHERF1 expression substantially increased apoptosis in PC-3M cells.

Influence of NHERF1 knockdown on cell cycle in PC-3M cells. Next, the effect of NHERF1 knockdown on the cell cycle was analyzed. Transfection with NHERF1 shRNA significantly increased the sub-G1 fraction (the fraction of apoptotic cells) compared with the luciferase shRNA-transfected and parental control cells $(22,10$ and $8 \%$ in NHERF1 shRNA-transfected, luciferase shRNA-transfected and parental cells, respectively; data not shown). Collectively, these results suggest that NHERF1 is important in cell growth, as well as cell cycle progression, in a subset of prostate cancer cell lines.

Influence of NHERF1 knockdown on the expression of apoptosis-associated proteins in PC-3M cells. As shown in Fig. 5, the expression of Bax, a pro-apoptotic protein, was unchanged 
following transfection with NHERF1 shRNA, while the expression of Bcl-2, an anti-apoptotic protein, was markedly decreased $(\mathrm{P}<0.05)$. These data supported the results of flow cytometry analysis indicating that apoptosis was increased following knockdown of NHERF1.

\section{Discussion}

The present study examined the effects of NHERF1 knockdown on the malignant phenotype in $\mathrm{PC}-3 \mathrm{M}$ prostate cancer cells. The results demonstrated that $N H E R F 1$ knockdown inhibited proliferation and migration, while increasing apoptosis. Therefore, NHERF1 may represent a novel oncogene in prostate cancer.

NHERF1 has been reported to be involved in the development and occurrence of cancer. Of note, abnormalities in NHERF1 expression have been demonstrated to be associated with the occurrence, development and metastasis of certain types of cancer $(4,9,10)$. While the details of these mechanisms remain to be fully elucidated, NHERF1 was reported to form a protein complex with epidermal growth factor receptor (EGFR) and neurofibromatosis type 2 (NF2) at intercellular adherens junctions (11), thereby mediating the internalization and signal transduction of EGFR in order to regulate various oncogenic processes. In addition, NHERF1 was reported to interact with platelet-derived growth factor receptor (PDGFR) to form a transduction complex with NF2 (12); it was suggested that this complex functions to block the signaling associated with PDGFR, thereby affecting apoptosis during the epithelial-to-mesenchymal transition and promoting cancer development and metastasis (13). In addition, NHERF1 was reported to interact with several other specific growth factor receptors that have important functions in proliferation, invasion and angiogenesis $(14,15)$. Therefore, NHERF1 was thought to directly or indirectly affect the development and progression of cancer (5).

The expression level of NHERF1 in prostate cancer varies depending on the pathological pattern and cancer phase (6) This may be explained by the observation that different cell systems and tissues have different genetic backgrounds. In addition, in samples where NHERF1 is expressed at similar levels, NHERF1 may combine with different proteins and thus exhibit different functions in samples with varying genetic backgrounds. In the prostate cancer cell system adopted in the present study (PC-3M cells), NHERF1 exhibited high endogenous expression, which was reduced through stable transfection with a NHERF1-targeting shRNA. The results indicated that inhibition of NHERF1 expression attenuated the proliferation and migration rates of PC-3M cells. Of note, major changes in cell cycle distribution were not observed (data not shown), although changes were detected in the height of the sub-G1 peak in flow cytometry analysis, which prompted the investigation of apoptosis in NHERF1-knockdown cells. It was then demonstrated that knockdown of NHERF1 increased apoptosis, thereby contributing to the observed inhibition of cell proliferation. Cell apoptosis, proliferation and differentiation are all basic, vital functions of cells, which are closely associated; imbalances in these functions have been associated with tumor development $(16,17)$. In general, apoptosis has a negative regulatory role in cancer cells and may inhibit tumor growth (18). Consistent with the increase in apoptosis observed in the present study, inhibition of $\mathrm{Bcl}-2$ expression in NHERF1 knockdown cells was detected. Bcl-2 is an anti-apoptotic protein; thus, inhibition of Bcl-2 expression may promote apoptosis. Therefore, in the present study, data from western blot analysis of apoptosis-associated proteins supported the flow cytometry results, demonstrating the role of NHERF1 in modulating the cell proliferation and apoptosis of prostate cancer cells.

In conclusion, the results of the present study demonstrated that NHERF1 was involved in regulating malignant biological behaviors, including proliferation and migration, in PC-3M cells. In addition, NHERF1 knockdown was demonstrated to promote apoptosis through inhibition of $\mathrm{Bcl}-2$ expression. Therefore, these results indicated that NHERF1 acts as an oncogenic protein in prostate cancer and may be a potential therapeutic target for the treatment of prostate cancer. Although these data support the role of NHERF1 in complexes with growth factor receptors and ligands or in influencing the expression and stability of $\beta$-catenin (19), further studies with other associated transmembrane ligands are required.

\section{Acknowledgements}

The present study was supported by the Basic Clinical Research Cooperation Project of Capital Medical University (Beijing, China; grant nos. 13JL20 and 13JL69), the Project of Beijing Obstetrics and Gynecology Hospital, Capital Medical University (grant no. 201208) the National Natural Science Foundation of the People's Republic of China (grant no. 81372739) and the Importation and Development of High-Caliber Talents Project of Beijing Municipal Institutions (grant nos. CIT\&TCD201304187 and CIT\&TCD201304190).

\section{References}

1. Jácome-Pita F, Sánchez-Salas R, Barret E, Amaruch N, Gonzalez-Enguita $\mathrm{C}$ and Cathelineau X: Focal therapy in prostate cancer: The current situation. Ecancermedicalscience 8: 435, 2014

2. Roudier MP, True LD, Higano CS, Vesselle H, Ellis W, Lange P and Vessella RL: Phenotypic heterogeneity of end-stage prostate carcinoma metastatic to bone. Hum Pathol 34: 646-653, 2003.

3. Georgescu MM, Cote G, Agarwal NK and White CL III: NHERF1/EBP50 controls morphogenesis of 3D colonic glands by stabilizing PTEN and ezrin-radixin-moesin proteins at the apical membrane. Neoplasia 16: e1-e2, 2014.

4. Takahashi Y, Morales FC, Kreimann EL and Georgescu MM: PTEN tumor suppressor associates with NHERF proteins to attenuate PDGF receptor signaling. EMBO J 25: 910-920, 2006.

5. Molina JR, Agarwal NK, Morales FC, Hayashi Y, Aldape KD, Cote $\mathrm{G}$ and Georgescu MM: PTEN, NHERF1 and PHLPP form a tumor suppressor network that is disabled in glioblastoma. Oncogene 31: 1264-1274, 2012.

6. Bartholow TL, Becich MJ, Chandran UR and Parwani AV: Immunohistochemical analysis of ezrin-radixin-moesin-binding phosphoprotein 50 in prostatic adenocarcinoma. BMC Urol 11: 12, 2011.

7. Prajapati A, Gupta S, Mistry B and Gupta S: Prostate stem cells in the development of benign prostate hyperplasia and prostate cancer: Emerging role and concepts. Biomed Res Int 2013: 107954, 2013.

8. International Organization for Standardization (ISO): ISO 10993-5: Biological evaluation of medical devices - Part 5: Tests for in vitro cytotoxicity. ISO, Geneva 2009

9. Malfettone A, Saponaro C, Paradiso A, Simone G and Mangia A: Peritumoral vascular invasion and NHERF1 expression define an immunophenotype of grade 2 invasive breast cancer associated with poor prognosis. BMC Cancer 12: 106, 2012.

10. Bellizzi A, Malfettone A, Cardone RA and Mangia A: NHERF1/EBP50 in Breast Cancer: Clinical Perspectives. Breast Care (Basel) 5: 86-90, 2010. 
11. Curto M, Cole BK, Lallemand D, Liu CH and McClatchey AI: Contact-dependent inhibition of EGFR signaling by Nf2/Merlin. J Cell Biol 177: 893-903, 2007.

12. James MF, Beauchamp RL, Manchanda N, Kazlauskas A and Ramesh V: NHERF binding site links the betaPDGFR to the cytoskeleton and regulates cell spreading and migration. J Cell Sci 117: 2951-2961, 2004.

13. Malavaki CJ, Roussidis AE, Gialeli C, Kletsas D, Tsegenidis, et al: Imatinib as a key inhibitor of the platelet-derived growth factor receptor mediated expression of cell surface heparan sulfate proteoglycans and functional properties of breast cancer cells. FEBS J 280: 2477-2489, 2013.

14. Yao W, Feng D, Bian W, Yang L, Li Y, Yang Z, Xiong Y, Zheng J, Zhai R and He J: EBP50 inhibits EGF-induced breast cancer cell proliferation by blocking EGFR phosphorylation. Amino Acids 43: 2027-2035, 2012

15. Jiang Y, Wang S, Holcomb J, Trescott L, Guan X, Hou Y, Brunzelle J, Sirinupong N, Li C and Yang Z: Crystallographic analysis of NHERF1-PLC $\beta 3$ interaction provides structural basis for CXCR2 signaling in pancreatic cancer. Biochem Biophys Res Commun 446: 638-643, 2014
16. Mamlouk S, Kalucka J, Singh RP, Franke K, MuschterA, Langer A, Jakob C, Gassmann M, Baretton GB and Wielockx B: Loss of prolyl hydroxylase-2 in myeloid cells and T-lymphocytes impairs tumor development. Int J Cancer 134: 849-858, 2014.

17. Chen Q, Lu HS, Gan MF, Chen LX, He K, Fan GM and Cao XQ: Expression and prognostic role of MEKK3 and pERK in patients with renal clear cell carcinoma. Asian Pac J Cancer Prev 16: 2495-2499, 2015.

18. Tatsuta T, Sugawara S, Takahashi K, Ogawa Y, Hosono M and Nitta K: Cancer-selective induction of apoptosis by leczyme. Front Oncol 4: 139, 2014.

19. Kreimann EL, Morales FC, de Orbeta-Cruz J, Takahashi Y, Adams $\mathrm{H}$, et al: Cortical stabilization of beta-catenin contributes to NHERF1/EBP50 tumor suppressor function. Oncogene 26: 5290-5299, 2007. 